\title{
A Web-Based Telemanagement System for Improving Disease Activity and Quality of Life in Patients With Complex Inflammatory Bowel Disease: Pilot Randomized Controlled Trial
}

Javier Del Hoyo ${ }^{1 *}$, MD; Pilar Nos ${ }^{1,2,3}, \mathrm{MD}$, PhD; Raquel Faubel ${ }^{4,5}, \mathrm{PhD}$; Diana Muñoz ${ }^{1}, \mathrm{RN}$; David Domínguez ${ }^{6}, \mathrm{CS}$; Guillermo Bastida $^{1,2,3}$, MD, PhD; Bernardo Valdivieso ${ }^{5,7,8}$, MD, PhD; Marisa Correcher ${ }^{9}$, PhD; Mariam Aguas ${ }^{1,2,3^{*}}$, $\mathrm{MD}, \mathrm{PhD}$

\footnotetext{
${ }^{1}$ Gastroenterology Department, La Fe University and Polytechnic Hospital, Valencia, Spain

${ }^{2}$ CIBEREHD (Networked Biomedical Research Center for Hepatic and Digestive Diseases), Valencia, Spain

${ }^{3}$ Health Research Institute La Fe, Valencia, Spain

${ }^{4}$ Physiotherapy Department, University of Valencia, Valencia, Spain

${ }^{5}$ Joint Research Unit in Biomedical Engineering (eRPSS: IIS La Fe-UPV), Valencia, Spain

${ }^{6}$ Connected Health Services, Valencia, Spain

${ }^{7}$ Home Care and Telemedicine Department, La Fe University and Polytechnic Hospital, Valencia, Spain

${ }^{8}$ Red de Investigación en Servicios de Salud en Enfermedades Crónicas (REDISSEC), Madrid, Spain

${ }^{9}$ Systems Department, La Fe University and Polytechnic Hospital, Valencia, Spain

these authors contributed equally
}

\section{Corresponding Author:}

Mariam Aguas, MD, PhD

Gastroenterology Department

La Fe University and Polytechnic Hospital

Av Fernando Abril Martorell 106

Valencia, 46026

Spain

Phone: 34600859948

Fax: 349612462

Email: m.aguas.peris@gmail.com

\section{Abstract}

Background: The reported efficacy of telemedicine in patients with inflammatory bowel disease (IBD) is inconsistent among studies, and data for complex IBD are lacking.

Objective: We aimed to evaluate the impact of remote monitoring using a Web system-Telemonitorización de la Enfermedad de Crohn y Colitis Ulcerosa or Telemonitoring of Crohn's Disease and Ulcerative Colitis (TECCU)—as compared to standard care and telephone care on health outcomes and health care in patients with complex IBD.

Methods: We performed a 3-arm randomized controlled trial. Adult patients with IBD who received immunosuppressants and biological agents were recruited from the IBD Unit of a tertiary university hospital. The patients were randomized into groups to receive remote monitoring $\left(G_{-}\right.$TECCU), nurse-assisted telephone care $\left(G \_N T\right)$, or standard care with in-person visits $\left(G \_c o n t r o l\right)$. All patients completed the study visits at baseline and at 12 and 24 weeks in addition to each type of intervention. The primary outcome was the percentage of patients in remission at 24 weeks. Secondary health outcomes were quality of life, medication adherence, adverse effects, satisfaction, and social activities. Data on the number of outpatient visits and telephone calls, emergency visits, hospitalizations, IBD-related surgeries, and corticosteroid courses were also collected.

Results: A total of 63 patients were selected (21 patients in each group). During the study, 90.5\% (19/21) of patients in G_control, 95.2\% (20/21) in G_NT, and 85.7\% (18/21) in G_TECCU were compliant to the intervention. After 24 weeks, the percentage of patients in remission was higher in G_TECCU $(17 / 21,81 \%)$ than in G_NT $(14 / 21,66.7 \%)$ and G_control $(15 / 21,71.4 \%)$. A higher improvement in disease activity was observed in G_TECCU than in G_control in terms of the Harvey-Bradshaw/Mayo (odds ratio $=0.12,95 \% \mathrm{CI}=0.003-2.162, P=.19$ ) and Harvey-Bradshaw/Walmsley (odds ratio $=0.11,95 \% \mathrm{CI}=0.004-1.55, P=.13$ ) indexes. Improvement in disease activity was associated with a larger reduction in fecal calprotectin values in G_TECCU compared 
to $\mathrm{G}$ _control (estimated intervention effect: odds ratio=-0.90; $95 \% \mathrm{CI}=-1.96$ to $0.16, P=.11$ ). All completers adhered to treatment in G_TECCU. In addition, the quality of life, social activities, and satisfaction improved in all 3 groups. Although the number of outpatient visits and telephone calls was lower in G_TECCU than in G_NT and G_control, the safety profile was similar in all 3 groups.

Conclusions: This pilot clinical trial suggests that the TECCU Web-based system is a safe strategy for improving health outcomes in patients with complex IBD and reducing the use of health care resources.

Trial Registration: ClinicalTrials.gov NCT02943538; https:/clinicaltrials.gov/ct2/show/NCT02943538 (Archived by WebCite at http://www.webcitation.org/746CRRtDN).

(J Med Internet Res 2018;20(11):e11602) doi: $\underline{10.2196 / 11602}$

\section{KEYWORDS}

Crohn disease; e-health; inflammatory bowel disease; information and communication technology; telemedicine; ulcerative colitis

\section{Introduction}

Inflammatory bowel disease (IBD), comprising ulcerative colitis (UC) and Crohn disease (CD), is a chronic relapsing disorder characterized by inflammation of the gastrointestinal tract. Its natural progression includes flares and periods of remission, and IBD requires continuous and personalized follow-up to ensure long-term remission. Patients with IBD use health care resources significantly more often than patients with other conditions [1]. Importantly, 30\%-45\% of adult patients with IBD are nonadherent to treatment [2], which increases the probability of relapse by 4 -fold and consequently increases the health care costs [3]. The high percentage of nonadherence is associated with behavioral and psychological factors and the physician-patient relationship [2]. In addition, IBD is related to high levels of school absenteeism and work disability [4], interference with social activities, and impairment of health-related quality of life (HRQoL) [5]. Therefore, IBD has a significant medical, social, and financial impact.

To address these difficulties, telemedicine uses information and communication technologies (ICTs) to provide health care services remotely. Despite the heterogeneous nature and progress of IBD, ICTs allow tailored follow-up with better communication between physicians and patients [6-8] and provide educational resources that promote patient empowerment [6] and optimize treatment $[9,10]$. ICTs were initially used to support the management of other chronic diseases such as congestive heart failure [11], diabetes mellitus [12], and chronic obstructive pulmonary disease [13] and showed excellent acceptance by patients and improvement in HRQoL. Owing to these positive results, telemedicine systems have been evaluated in patients with IBD $[14,15]$.

A ground-breaking evaluation of telemedicine in patients with IBD by Cross et al. [16], who designed the Home Automated Telemanagement system, was acceptable, increased patients' knowledge about their disease, and facilitated greater self-control of IBD symptoms [17]. However, in a randomized controlled trial of 47 patients with UC in remission or with mild UC activity, no significant differences were noted in disease activity, HRQoL, or medication adherence between patients who followed the telemedicine system and those who received usual care at 12 months [18]. These results were partially explained by a higher discontinuation rate in the Home
Automated Telemanagement group due to the platform design. To avoid similar problems, the authors subsequently designed a mobile phone-based Web program to determine the impact of telemedicine on health outcomes [19]. Web programs and mHealth interventions are increasingly used to provide information and health care at a distance. Unlike other telehealth systems, Web-based apps do not require home installation and are less expensive. Web-based systems adapted to IBD are safe and feasible for adults and adolescents [20-22], improve medication adherence, and reduce the duration of relapses [6]. A recent multicenter, randomized controlled trial including more than 900 patients with different subtypes of IBD showed that compared with standard care, empowerment through these systems reduces outpatient visits and hospital admissions, with potential cost savings [8]. Web-based systems are currently the most useful platforms, and their application allows development of distance care projects aimed at providing access to health care in remote areas [23,24]. These models promote collaboration and knowledge exchange between specialists, potentially reducing variability in clinical practice, and could modify the future structure of health systems if they prove to be cost-effective. However, the effect of telemedicine systems on disease outcomes is inconsistent and varies across studies according to the population and health care system in which they are applied $[6,8,18,20,22]$. Moreover, no specific studies have thus far focused on the impact of telemedicine in patients with complex IBD.

Our research group designed a Web-based telemanagement system known as Telemonitorización de la Enfermedad de Crohn y Colitis Ulcerosa or Telemonitoring of Crohn's Disease and Ulcerative Colitis (TECCU) for remote monitoring of patients with moderate-to-severe IBD activity or who initiate treatment with corticosteroids, immunosuppressants, and biological agents. We performed a randomized controlled trial to assess the safety of care provided through this system and its impact on disease activity, HRQoL, medication adherence, satisfaction, work productivity, social activities, and use of health care resources and compared these outcomes with those of nurse-assisted telephone care and standard face-to-face visits. 


\section{Methods}

\section{Study Design}

A 3-arm, parallel-group, randomized controlled trial was performed to compare the impact of the Web-based telemanagement system TECCU, nurse-assisted telephone care, and standard face-to-face visits on health outcomes and outpatient visits in patients with complex IBD. Neither the patients nor the researchers were masked to the intervention, but the results were analyzed by an independent statistician who was blinded to group identification.

\section{Ethical Considerations}

The study protocol was reviewed and approved by the local independent ethics committee of $\mathrm{La} \mathrm{Fe}$ University and Polytechnic Hospital, Valencia, Spain; the regional independent ethics committee (Comité Ético Autonómico de Estudios Clínicos de Medicamentos y Productos Sanitarios de la Comunitat Valenciana); and the Spanish Agency of Medicines and Medical Devices (Agencia Española de Medicamentos y Productos Sanitarios). According to the physicians involved in the study, the risks did not outweigh the potential benefits, and each participant provided informed consent without coercion before inclusion in the study. The trial is registered at ClinicalTrials.gov with the identifier NCT02943538.

\section{Patient Selection}

Patients were recruited from the IBD Unit of a tertiary university hospital. All participants were diagnosed with IBD at least 6 months previously and according to the internationally accepted criteria $[25,26]$.

\section{Inclusion and Exclusion Criteria}

The inclusion criteria were age $\geq 18$ years; initiation of therapy with corticosteroids, immunosuppressants, and biological agents due to disease activity; and provision of written informed consent to participate in the study. The exclusion criteria were inability to speak and read Spanish; inability to manage a mobile phone or tablet or the Internet or not having a telephone line; participation in other clinical trials during the inclusion period; uncontrolled medical or psychiatric disease; presence of ileorectal or ileal pouch-anal anastomosis; receipt of definitive ileostomy; perianal disease; and pregnancy.

\section{Recruitment}

This project was funded in 2012, and the platform was installed and configured in 2013-2014. Enrollment was started in October 2014 and ended in June 2016. The follow-up ended in December 2016. Patients were included consecutively from the Outpatient Clinic of the IBD Unit or the Gastroenterology Department if they were admitted for a flareup of IBD. During the visit, the inclusion and exclusion criteria were verified, and the patient was informed about the study through the patient-information sheet. If the patient agreed to participate, he or she signed the informed consent document.

\section{Randomization}

Eligible patients were randomized to 1 of the 3 groups to receive remote monitoring $\left(\mathrm{G}_{-} \mathrm{TECCU}\right)$, nurse-assisted telephone care
(G_NT), or standard care with in-person visits (G_control) in a $1: 1: 1$ ratio by using a block randomization method through a Web-based tool [27] in order to generate a random-allocation sequence and ensure allocation concealment. Once a number was assigned, it could not be reassigned, and members of the research team who were in contact with patients did not have access to the randomization tables or lists.

\section{Study Outcomes}

Participants completed study visits at the baseline, 12 weeks, and 24 weeks, in addition to routine visits scheduled for their clinical care. The variables measured at baseline were sociodemographic variables, disease profile and activity, HRQoL, adverse events, medication adherence, and patient satisfaction.

\section{Primary Outcome}

The primary objective of the study was to determine the percentage of patients in clinical remission at 24 weeks. Remission was evaluated using the modified Harvey-Bradshaw index (HBI) for patients with CD [28]. For patients with UC, we used the Simple Clinical Colitis Activity Index (SCCAI, also known as the Walmsley index) [29] for remote checkups together with the partial Mayo score for face-to-face visits [30].

Patients with $\mathrm{CD}$ and an $\mathrm{HBI}<5$ were considered to be in clinical remission, whereas patients with scores of 5-7, 8-16, or $>16$ were considered to have mild, moderate, or severe activity, respectively [28]. For remote checkups in patients with $\mathrm{UC}$, clinical remission was defined as a Walmsley score $\leq 2$, whereas mild-to-moderate and severe activities were defined as scores of 3-5 and $>5$, respectively [31]. In the face-to-face visits, clinical remission was defined as a partial Mayo score $\leq$ 2 and no individual Mayo subscore > 1; scores of 2-5, 6-8, and 9 were defined as mild, moderate, and severe disease activity, respectively [30].

Laboratory parameters were measured at baseline and each subsequent visit according to individual patient's schedule and included a complete blood analysis with nutritional profile and C-reactive protein level $(\mathrm{mg} / \mathrm{L})$. Fecal calprotectin $(\mathrm{FC}, \mu \mathrm{g} / \mathrm{g}$ ) was assessed at baseline, 12 weeks, and 24 weeks after inclusion. Changes in the medication were made on the basis of the results obtained, in association with clinical activity indexes, and were prescribed according to specific intervention plans adapted to the severity of each alert.

\section{Secondary Outcomes}

The HRQoL of patients at inclusion and at week 24 was evaluated using the specific questionnaire Inflammatory Bowel Disease Questionnaire 9 (IBDQ-9) and the generic questionnaire EuroQol-5D (EQ-5D). IBDQ-9 is a validated questionnaire comprising 9 items distributed in 4 dimensions: bowel symptoms, systemic symptoms, emotional function, and social function. It correlated very well with the Spanish version of the 36-item IBDQ [32]. Each item is scored on a 7-point Likert scale, with an overall score ranging from 7 (lowest QoL) to 63 (highest QoL) points, and calculated over 100 points. EQ-5D is a generic questionnaire that has been used for patients with various chronic diseases such as IBD and was previously 
validated in Spain [33]. This instrument provides a global value for HRQoL, with 5 questions related to 5 dimensions: mobility, selfcare, usual activities, pain or discomfort, and anxiety or depression. Additionally, a visual analog scale (VAS) was used. Each question was scored with 1, 2, or 3 points, depending on whether impairment in the dimension assessed was nonexistent, moderate, or extreme, respectively; subsequently, the results were converted for each dimension according to the specific coefficients calculated for the Spanish population [34]. The maximum score of 1 represents the best health status, a score of 0 represents a health state considered equivalent to death, and negative index scores represent health states considered worse than death. The VAS rates overall health between 0 (poorest imaginable health) and 100 (best imaginable health).

We assessed the impact of disease on work productivity and activities of daily living using the Work Productivity and Activity Impairment (WPAI) questionnaire [35], which patients completed at baseline and week 24 . The questionnaire comprises 6 questions on the effect of the disease on work and activities of daily living during the previous 7 days. A higher score on the questionnaire indicates a more pronounced effect on work and daily activities. The Spanish version has been validated and is reproducible in patients with $\mathrm{CD}$.

Medication adherence was evaluated using the Morisky-Green index [36], which has been used to evaluate adherence in patients with IBD in clinical trials [18]. We considered adherence to be adequate when the patient answered all questions correctly and inadequate if any answer was associated with nonadherence.

We recorded the number of outpatient visits and telephone consultations, as registered in the NOMHADCHRONIC app (version V2.RC6; Connected Health Services SL, Valencia, Spain) for patients in G_TECCU and in the Orion Clinic information system (as part of daily clinical practice at our hospital) for all 3 groups during the study. Patients who adhered to $>80 \%$ of checkups in the study protocol were considered to be compliant.

The safety of each intervention was assessed by measuring the number of visits to the emergency department, hospitalizations, IBD-related surgeries, corticosteroid courses, and adverse effects to medication. Finally, patient satisfaction with the care received was evaluated at 24 weeks by using an adapted version of the Client Satisfaction Questionnaire, which comprises 6 questions (measured on a scale of 0-10) on the quality, usefulness, and viability of the system of care applied for each case [37].

\section{Setting and Interventions}

This pilot trial was developed at the tertiary referral center La Fe University and Polytechnic Hospital, Valencia, Spain. The hospital serves more than 1500 patients with IBD, has 2 specialist IBD nurses, and provides an email and telephone consultation structure for IBD patients to contact the hospital.

The trial had 3 arms and compared remote monitoring through a Web-based telemanagement system, nursing care by telephone, and usual care provided in our IBD Unit (Outpatient Clinic). All patients completed the study visits at baseline and 12 and 24 weeks, in addition to routine visits to the IBD clinic, telephone consultations, or Web telemonitoring based on group assignment at randomization. Disease activity, HRQoL, adverse effects, adherence, and use of health care resources were measured at baseline and during the 24-week follow-up.

To adequately control disease activity and adverse effects of immunosuppressants, patients treated with these drugs alone or in combination with biological agents were monitored every 1-2 weeks during the first month, every 2-4 weeks between months 1 to 3 , and every 4 weeks from month 3 until the end of follow-up. Patients treated with biological agents alone were monitored every 2-4 weeks during the entire follow-up period. Patients from all 3 arms who took the same type of drug underwent these follow-up schedules; the schedules only differed in terms of the monitoring: patients in G_TECCU were monitored via the NOMHADCHRONIC app, those in G_NT were monitored via a telephone line managed by nursing staff from the IBD Unit, and those in G_control were monitored by the usual face-to-face visits combined with telephone calls made by physicians according to standard clinical practice. Moreover, additional clinical visits were performed, if required, during patient evolution in any of the 3 arms.

\section{Telemonitoring Apps}

In G_TECCU, follow-up and monitoring were performed telematically using the integrated platform for management of chronically ill patients (NOMHADCHRONIC app), which was designed to meet the specific needs of this group. The NOMHADCHRONIC platform is an innovative technological system that was designed to boost the rollout of services for the management of chronically ill patients. The telemanagement care system was developed in collaboration with patients, the La Fe telemedicine Unit, and Tecnologías para la Salud y el Bienestar SA. The protocol of the Web-based telemanagement system (TECCU) has been described elsewhere [27]. TECCU is a secure webpage with an HTTP app for mobile phones, tablets, and computers. Patients connected to the platform via the Internet using a computer or an app on a mobile phone or tablet had to self-complete questionnaires. In addition, they received advice, reminders, educational material about their disease, and information on prevention. This information was received by the case managers and filtered using an intelligent prioritization system with generation of alerts and push notifications according to an integrated intervention protocol.

Patients monitored via the TECCU used NOMHADhome [38]. The resources of the NOMHADhome platform were also available on the NOMHAD mobile app, which patients could download onto their mobile phones. After inclusion, these patients automatically received an email with a personal code that allowed them to access the platform. Each patient profile contained the following information: contact information, active IBD medications, testing schedule (blood and stool tests), log of disease activity, medication use, body weight, vital signs, HRQoL, alerts and action plans, progress of inflammatory activity and vital signs in the form of graphs, electronic messaging to the study nurse coordinator and health care provider, and educational tips.

Patients in G_TECCU had to answer several simple questions related to their IBD symptoms and possible adverse effects since 
the last evaluation via text messaging. In the main menu of the platform, patients accessed the questionnaires by clicking on specific icons designed for this purpose and could answer the multiple choice and yes or no questions needed to complete these items (Figures 1 and 2). We established individualized alerts and action plans based on the answers to questions about the activity index, adverse effects, and blood biochemistry results. A scale of values was assigned for each alert depending on the severity (green, yellow, orange, and red zone).

After receiving an alert, the specialized medical staff, in collaboration with the nurses, used the general recommendations of the action plans to guide medication adjustments in biological therapy, immunomodulators, corticosteroids, and salicilates. These treatment changes were performed with the support of the platform messaging system in combination with telephone calls or in-person visits when it was necessary to train patients for the administration of medications such as subcutaneous biological agents. Once the disease was in remission again (green zone), the patient had to continue with the initially programmed follow-up. All patients had access to tools that informed them about their disease. In G_TECCU, information was available on the NOMHADhome platform itself; in G_control and G_NT, patients received written documents with the same information.

Figure 1. Homepage of the NOMHADhome platform for access to questionnaires (patient version).
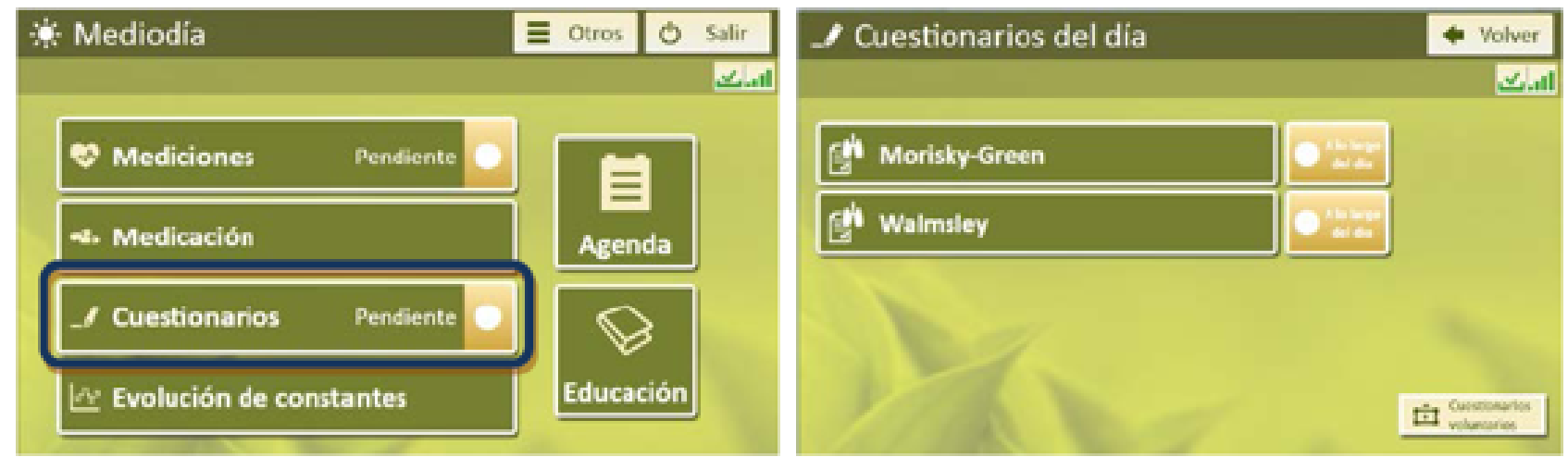

\section{성 Morisky-Green}

\section{Cuestionario de Morisky-Green}

¿Se olvida alguna vez de tomar el medicamento?

\section{O si}

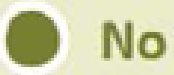

\section{Pregunta № 1}
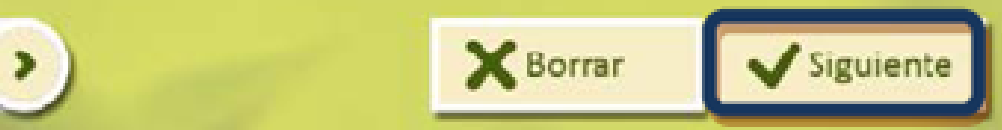
Figure 2. Home page of the NOMHADmobile platform for access to vital signs (patient version).

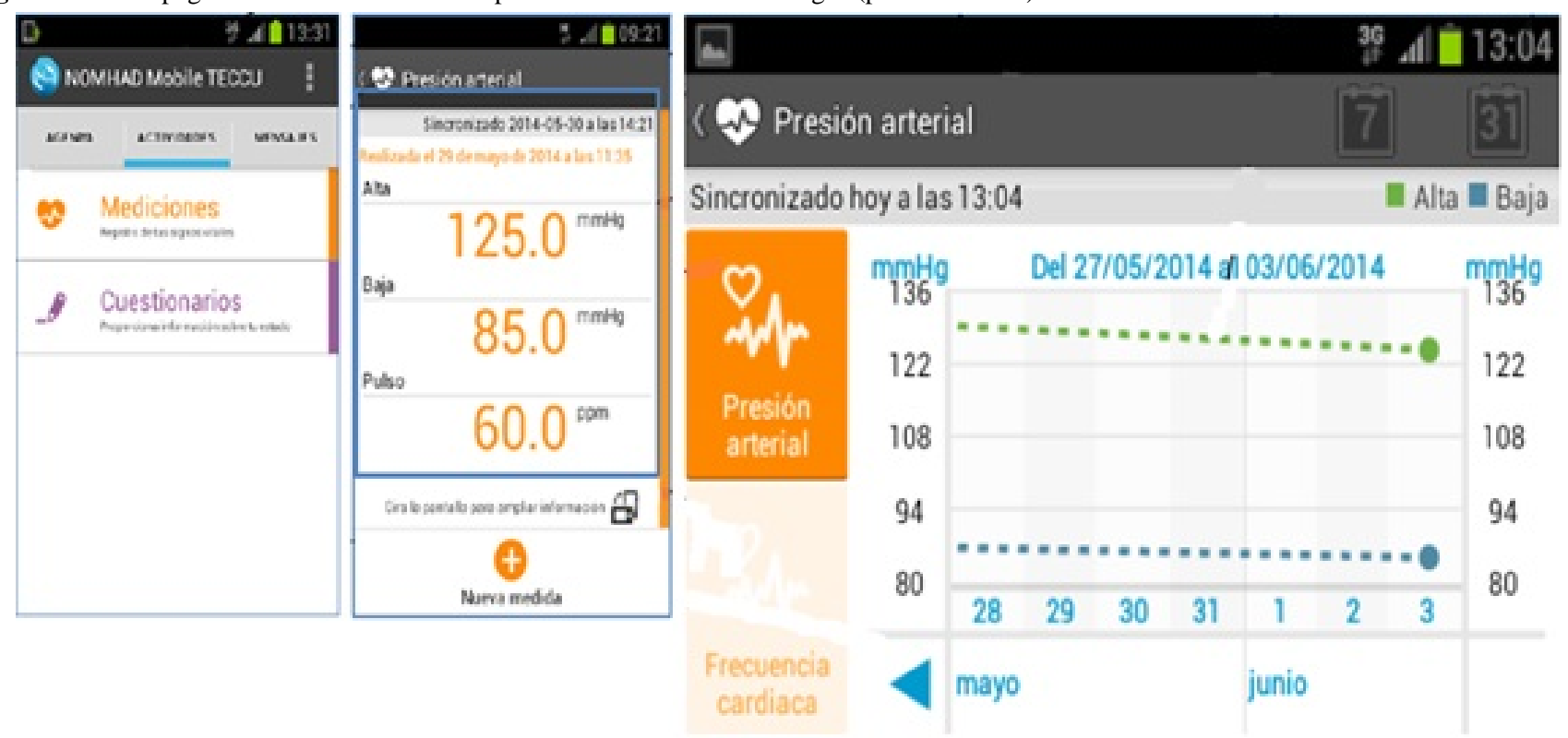

\section{Usual Care Provided in the Inflammatory Bowel Disease Unit}

The $\mathrm{G}_{-}$control patients received the normal care provided in the IBD Unit (Outpatient Clinic) for patients with moderately to highly complex IBD, based on national and European clinical guidelines $[25,26,39]$. Treatment was adjusted according to the evolution of disease activity and medication adherence, which was measured using specific indexes and biological markers used to report the study outcomes during office visits or telephone calls. In addition, to measure the time in remission, clinical activity of patients was self-recorded in a diary on paper at home weekly during the first 12 weeks and every 2 weeks subsequently, until the end of the follow-up.

This care was complemented by ad hoc hospital care in case of flareups or if the patient's health deteriorated for any reason. Ad hoc intensive care was maintained until the patient's condition stabilized, at which point he or she returned to follow-up based on standard care in the Unit. For comparability among the 3 groups, we provided information on all available on-paper educational materials about IBD for remotely monitored patients as well as information on prevention and written action plans in case of flareups. Questionnaires on HRQoL, satisfaction, and work productivity were recorded at baseline and at the end of the study.

\section{Nursing Care by Telephone}

The G_NT patients were asked about their health through telephone calls by the nursing staff in the IBD Unit. We performed telephone assessment periodically by using structured interviews to evaluate health status, and clinical activity was self-recorded at home, as described for the $G_{-}$control patients. The interventions depended on the results of the interview and changes in the medication or follow-up schedule established by nurses with the support of medical staff, according to the alerts and action plans designed in the intervention protocol [27]. Furthermore, we provided these patients with all educational elements made available to the other 2 groups.

\section{Notification of Adverse Effects}

TECCU is a minimum-risk system. The characteristics of the interventions in the present study are designed such that patients do not experience direct adverse effects. The interventions were proposed to control and achieve long remission periods in patients with IBD and were not expected to cause lesions or damage to the patients' health, since we did not test a new experimental drug. We evaluated a Web-based telemonitoring system designed to improve communication between patients and health care providers, with a proactive remote follow-up method, allowing changes in the patients' treatment and follow-up schedule when flareups are detected or when the flareups increase in severity as per the alert system. In addition, we incorporated educational elements in G_TECCU and the other 2 groups to make them more comparable.

With respect to the platform, a risk-minimization study was performed to ensure accomplishment of ethical norms and that the appropriate ethics committees approved the study. We also recorded all adverse events from the time the patient provided consent to participate in the study until 28 days after study completion.

\section{Power Calculation}

The most-efficient means of determining differences between the 3 groups was by contrasting differences in activity indexes for CD and UC. Considering the differences in the scales, the analysis was stratified by performing a comparison of patients with UC and another comparison of patients with CD. The sample size was calculated by estimating the size required to detect a difference of 3 points in the HBI; considering an SD of 4 , a power of $80 \%$, and an alpha significance level of .05 , a total of 30 patients with CD (10 per arm) was required. In addition, for a 2-point difference in the Mayo index, an SD of 2.5 , a power of $80 \%$, and an alpha significance level of .05 , a total of 30 patients with UC (10 per arm) was required. Therefore, the overall sample size was 60 patients (20 per arm). We also stratified patients globally (CD and UC) by comparing 
patients in remission or with inflammatory activity, irrespective of disease severity (mild, moderate, or severe).

\section{Statistical Analysis}

First, we described the characteristics of patients in the test and control groups by applying appropriate estimators (means, medians, or proportions) according to the type of variables and evaluated possible differences between the groups in the main and secondary outcomes by using regression models to assess differences in these measures. We evaluated the effects of the different treatment groups on the probability of remission using mixed-effects logistic regression models including an interaction between week and treatment groups. Individuals were included in the model as a random intercept to correct for the nonindependent data. Differences in slopes for weeks 12 and 24 were evaluated by assessing the interaction between week and treatment groups. $P$ values were estimated using the Satterthwaite approximation for degrees of freedom. We used mixed-effects ordinal logistic regression models to evaluate progress according to the IBDQ-9 and EQ-5D scores in the 3 study groups between weeks 0 and 24 . However, owing to convergence problems in assessing medication adherence (because of the reduced sample size and the ordinal character of the Morisky-Green test), Bayesian techniques were applied in the ordinal logistic regression model. Differences in the percentage of missed work hours, work impairment, and social impairment were analyzed using mixed-effects linear regression and mixed-effects beta regression models (\% work hours missed). Profile likelihood $95 \%$ confidence intervals were calculated for all estimations, and $P<.05$ was considered statistically significant. A likelihood-based analysis for all patients included was performed using $\mathrm{R}$ (version 3.5.1) with the lme4 (version 1.1-17), lmerTest (version 3.0-1), brms (version 2.2.0), clickR (version 0.4.04), ordinal (version 2018.4-19), and glmmADMB (0.8.3.3) packages [40].

\section{Results}

\section{Study Sample}

A total of 68 patients with complex IBD were invited to participate in this study between October 2014 and June 2016, of which $3(4.4 \%)$ declined to participate owing to inaccessibility to the Internet at home and $2(2.9 \%)$ did not meet the inclusion criteria. The remaining 63 eligible patients provided informed consent and were randomly assigned to the 3 groups (21 patients in each group, Figure 3). During the study period, all patients except 1 patient in G_TECCU (95.2\%) continued to use the Web-based telemanagement system and 2 other patients did not respond to $>80 \%$ of checkups. The remaining 18 patients $(85.7 \%)$ in G_TECCU showed good adherence to the study protocol as compared to 19 patients $(90.5 \%)$ in G_control and 20 patients $(95.2 \%)$ in G_NT. We did not observe any differences between the completers and patients who did not adhere to the study protocol. The baseline characteristics of the 3 study groups are shown in Table 1.

\section{Disease Activity}

According to the HBI and the partial Mayo scores, $47.6 \%$ (10/21), $38.1 \%(8 / 21)$, and $57.1 \%(12 / 21)$ of patients were in clinical remission at baseline in G_TECCU, G_NT, and G_control, respectively. The baseline percentage of remission according to the Walmsley score for UC was the same for each group and improved progressively at 12 and 24 weeks in all 3 groups. This percentage was higher in $\mathrm{G}_{-}$TECCU, even after considering dropouts, with $81 \%$ of patients (17/21) inactive after 24 weeks compared with $66.7 \%(14 / 21)$ in G_NT and $71.4 \%$ (15/21) in G_control (using both Mayo and Walmsley scores for evaluation of UC); therefore, the percentage of patients in remission increased by $33.4 \%$ in G_TECCU, $28.6 \%$ in G_NT, and $14.3 \%$ in G_control (Figure 4). In the intention-to-treat analysis, a higher probability of remission was observed in G_TECCU than in G_control at 12 weeks (odds ratio $(\mathrm{OR})=0.12,95 \% \mathrm{CI}=0.003-2.103, P=.18)$ and 24 weeks $(\mathrm{OR}=0.12,95 \% \mathrm{CI}=0.003-2.162, P=.19)$ using the HBI-Mayo scores. With the HBI-Walmsley scores, no differences were observed between G_TECCU and G_control at 12 weeks $(\mathrm{OR}=0.92,95 \% \mathrm{CI}=0.08-9.71, P=.94)$, but the probability of remission was higher in $G_{-}$TECCU at 24 weeks $(\mathrm{OR}=0.11$, $95 \% \mathrm{CI}=0.004-1.55, P=.13)$. The median time in remission was 17.9 weeks (interquartile range, 12-24 weeks) in G_TECCU compared to 17.3 weeks (interquartile range, 10-24 weeks) in G_NT and 14.3 weeks (interquartile range, 8-24 weeks) in G_control.

Disease activity was evaluated on the basis of FC levels throughout the study. At 24 weeks, the median FC level for clinical activity improved progressively from a baseline value of $490 \mu \mathrm{g} / \mathrm{g}$ to $137 \mu \mathrm{g} / \mathrm{g}$ in $\mathrm{G}_{-}$TECCU and from $526 \mu \mathrm{g} / \mathrm{g}$ to $115.5 \mu \mathrm{g} / \mathrm{g}$ in G_NT; however, this reduction was smaller in G_control, from $330 \mu \mathrm{g} / \mathrm{g}$ to $230 \mu \mathrm{g} / \mathrm{g}$. The improvement in FC levels was larger in G_TECCU than in G_control, with an estimated intervention effect of $-0.76(95 \% \overline{C I}=-1.85$ to 0.336 , $P=.18)$ in the reduction of $\mathrm{FC}$ values at 12 weeks and -0.90 $(95 \% \mathrm{CI}=-1.96$ to $0.16, P=.11)$ at 24 weeks. Similarly, a larger improvement was noted in $G_{-}$NT than in G_control at the same time points, with an estimated intervention effect of $-0.61(95 \%$ $\mathrm{CI}=-1.62$ to $0.39, P=.25)$ at 12 weeks and $-0.91(95 \% \mathrm{CI}=-1.96$ to $0.15, P=.10$ ) at 24 weeks (Figure 5). 
Figure 3. Flowchart of study participants. G_CONTROL: group receiving standard care with in-person visits; G_NT: group receiving nurse-assisted telephone care; G_TECCU: group receiving remote monitoring; IBD: inflammatory bowel disease.

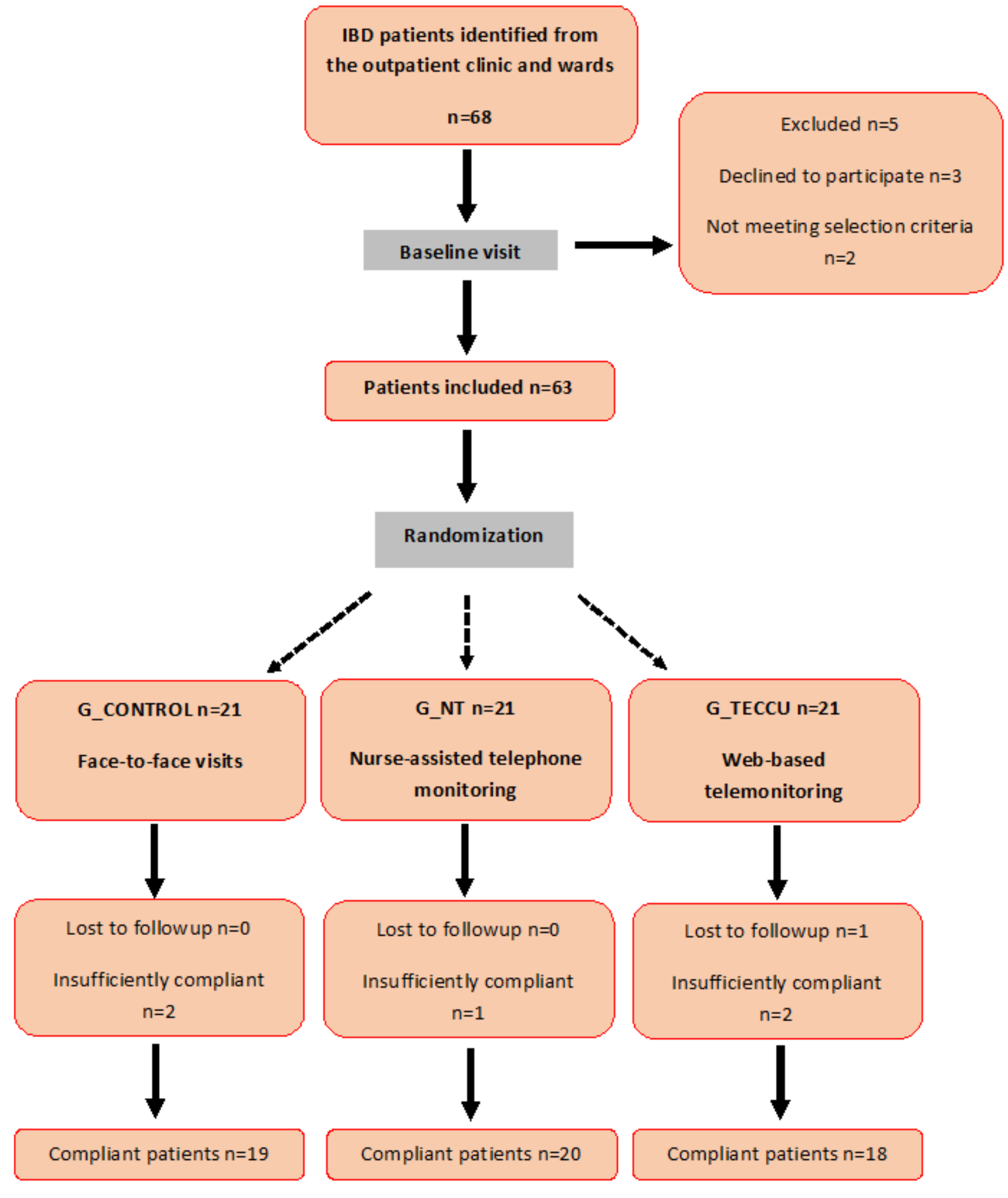

Patients analyzed at 12 and 24 weeks 
Table 1. Baseline characteristics of patients.

\begin{tabular}{|c|c|c|c|}
\hline Characteristics & Control group $(\mathrm{n}=21)$ & Telephone group $(\mathrm{n}=21)$ & TECCU $^{\mathrm{a}}$ group $(\mathrm{n}=21)$ \\
\hline Median age, years (range) & $39.31(22-61)$ & $40.91(24-60)$ & $41.32(19-66)$ \\
\hline \multicolumn{4}{|l|}{ Sex, n (\%) } \\
\hline Men & $12(57.1 \%)$ & $12(57.1 \%)$ & $9(42.9 \%)$ \\
\hline Women & $9(42.9 \%)$ & $9(42.9 \%)$ & $12(57.1 \%)$ \\
\hline \multicolumn{4}{|l|}{ Education, $n(\%)$} \\
\hline Primary & $4 / 21(19 \%)$ & $4 / 21(19 \%)$ & $5 / 21(23.8 \%)$ \\
\hline Secondary & $9 / 21(42.9 \%)$ & $6 / 21(28.6 \%)$ & $6 / 21(28.6 \%)$ \\
\hline University & $8 / 21(38.1 \%)$ & $11 / 21(52.4 \%)$ & $10 / 21(47.6 \%)$ \\
\hline \multicolumn{4}{|l|}{ Disease profile } \\
\hline Crohn disease, $\mathrm{n}(\%)$ & $14 / 21(66.7 \%)$ & $13 / 21(61.9 \%)$ & $13 / 21(61.9 \%)$ \\
\hline Ulcerative colitis, $\mathrm{n}(\%)$ & $7 / 21(33.3 \%)$ & $8 / 21(38.1 \%)$ & $8 / 21(38.1 \%)$ \\
\hline Median time since diagnosis, months (range) & $123.32(6-427)$ & $108.27(7-452)$ & $146.72(7-424)$ \\
\hline \multicolumn{4}{|l|}{ Treatment $(\%)$} \\
\hline Immunomodulators & $10 / 21(47.6 \%)$ & $10 / 21(47.6 \%)$ & $9 / 21(42.9 \%)$ \\
\hline Biological monotherapy & $4 / 21(19 \%)$ & $4 / 21(19 \%)$ & $4 / 21(19 \%)$ \\
\hline Combination therapy & $6 / 21(28.6 \%)$ & $5 / 21(23.8 \%)$ & $6 / 21(28.6 \%)$ \\
\hline Corticosteroids & $1 / 21(4.8 \%)$ & $2 / 21(9.5 \%)$ & $2 / 21(9.5 \%)$ \\
\hline Clinical Remission, n (\%) & $12 / 21(57.1 \%)$ & $8 / 21(38.1 \%)$ & $10 / 21(47.6 \%)$ \\
\hline Crohn disease & $10 / 14(71.4 \%)$ & $6 / 13(46.2 \%)$ & $9 / 13(69.2 \%)$ \\
\hline Ulcerative colitis & $2 / 7(28.6 \%)$ & $2 / 8(25 \%)$ & $1 / 8(12.5 \%)$ \\
\hline Median calprotectin level, $\mu \mathrm{g} / \mathrm{g}$ (interquartile range) & $330(103-617)$ & $526(115-1724)$ & $490(23-2016)$ \\
\hline \multicolumn{4}{|l|}{ Quality of life } \\
\hline Median IBDQ-9 $9^{\mathrm{b}}$ score (interquartile range) & $38.50(33.25-46.75)$ & $37.50(28.75-46.25)$ & $42.00(33.75-47.50)$ \\
\hline Median EQ-5D ${ }^{c}$ score (interquartile range) & $0.816(0.754-0.914)$ & $0.825(0.710-0.914)$ & $0.825(0.576-0.914)$ \\
\hline Median VAS ${ }^{\mathrm{d}}, \%$ (interquartile range) & $60.5 \%(50 \%-85 \%)$ & $62.5 \%(50 \%-80 \%)$ & $60 \%(40 \%-90 \%)$ \\
\hline Medication adherence, $\mathrm{n}(\%)$ & $14 / 21(66.7 \%)$ & $7 / 21(33,3 \%)$ & $12 / 21(57,1 \%)$ \\
\hline \multicolumn{4}{|l|}{ WPAI $^{\mathrm{e}}$} \\
\hline Not working, n (\%) & $8 / 21(38.1 \%)$ & $7 / 21(33.3 \%)$ & $5 / 21(23.8 \%)$ \\
\hline $\begin{array}{l}\text { Percentage of work hours missed, median (interquartile } \\
\text { range) }\end{array}$ & $27.5 \%(0 \%-52 \%)$ & $40 \%(15 \%-62.5 \%)$ & $32.5 \%(7.5 \%-57.5 \%)$ \\
\hline Work impairment score, median (interquartile range) & $7(2.75-10)$ & $7(3-10)$ & $10(2.25-10)$ \\
\hline Social impairment score, median (interquartile range) & $3.5(1-5.75)$ & $3.5(2-7)$ & $6(2.75-8)$ \\
\hline Satisfaction score, median (interquartile range) & $49.5(42.5-53.75)$ & $53(50-59)$ & $52(47.5-55)$ \\
\hline
\end{tabular}

'TECCU: Telemonitoring of Crohn's Disease and Ulcerative Colitis.

bIBDQ-9: Inflammatory Bowel Disease Questionnaire 9.

${ }^{c}$ EQ-5D: EuroQol-5D.

${ }^{\mathrm{d}}$ VAS: visual analog scale.

eWPAI: Work Productivity and Activity Impairment. 
Figure 4. Evolution of disease activity over the study period in the 3 groups. G_CONTROL: group receiving standard care with in-person visits; G_NT: group receiving nurse-assisted telephone care; G_TECCU: group receiving remote monitoring; HBI: Harvey-Bradshaw index.
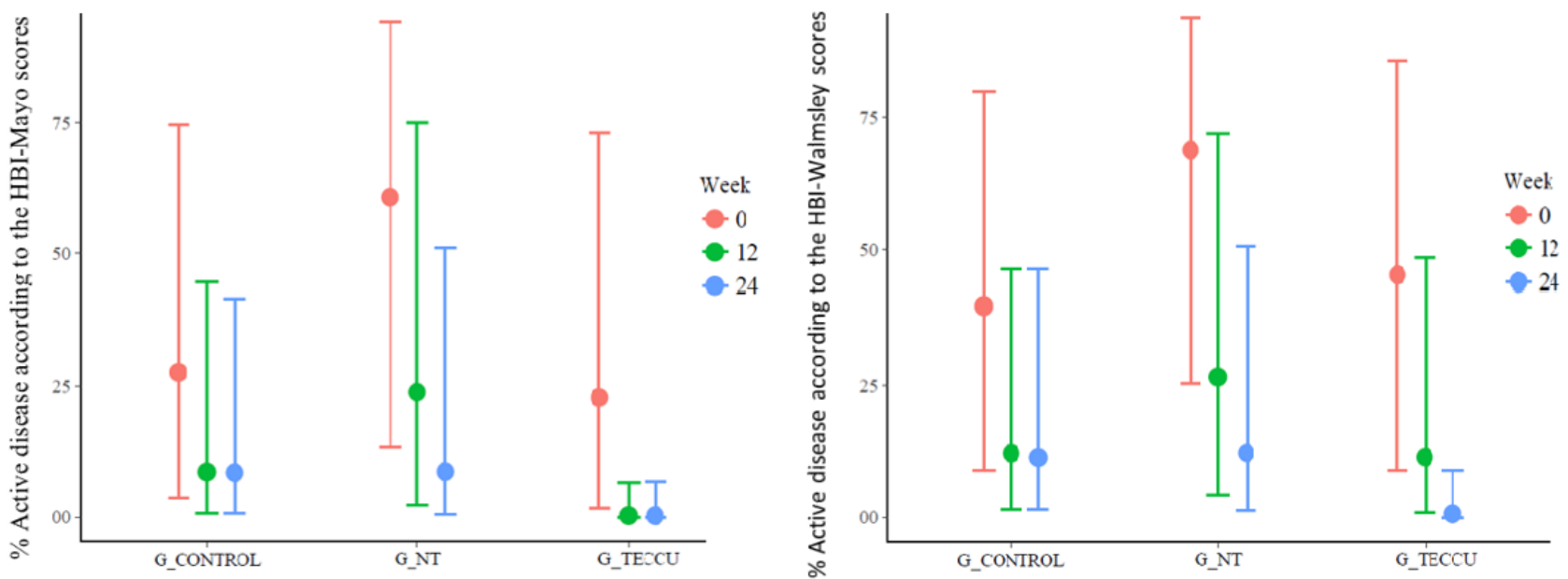

Figure 5. Evolution of the median fecal calprotectin levels over the study period in the 3 groups. G_CONTROL: group receiving standard care with in-person visits; G_NT: group receiving nurse-assisted telephone care; G_TECCU: group receiving remote monitoring.

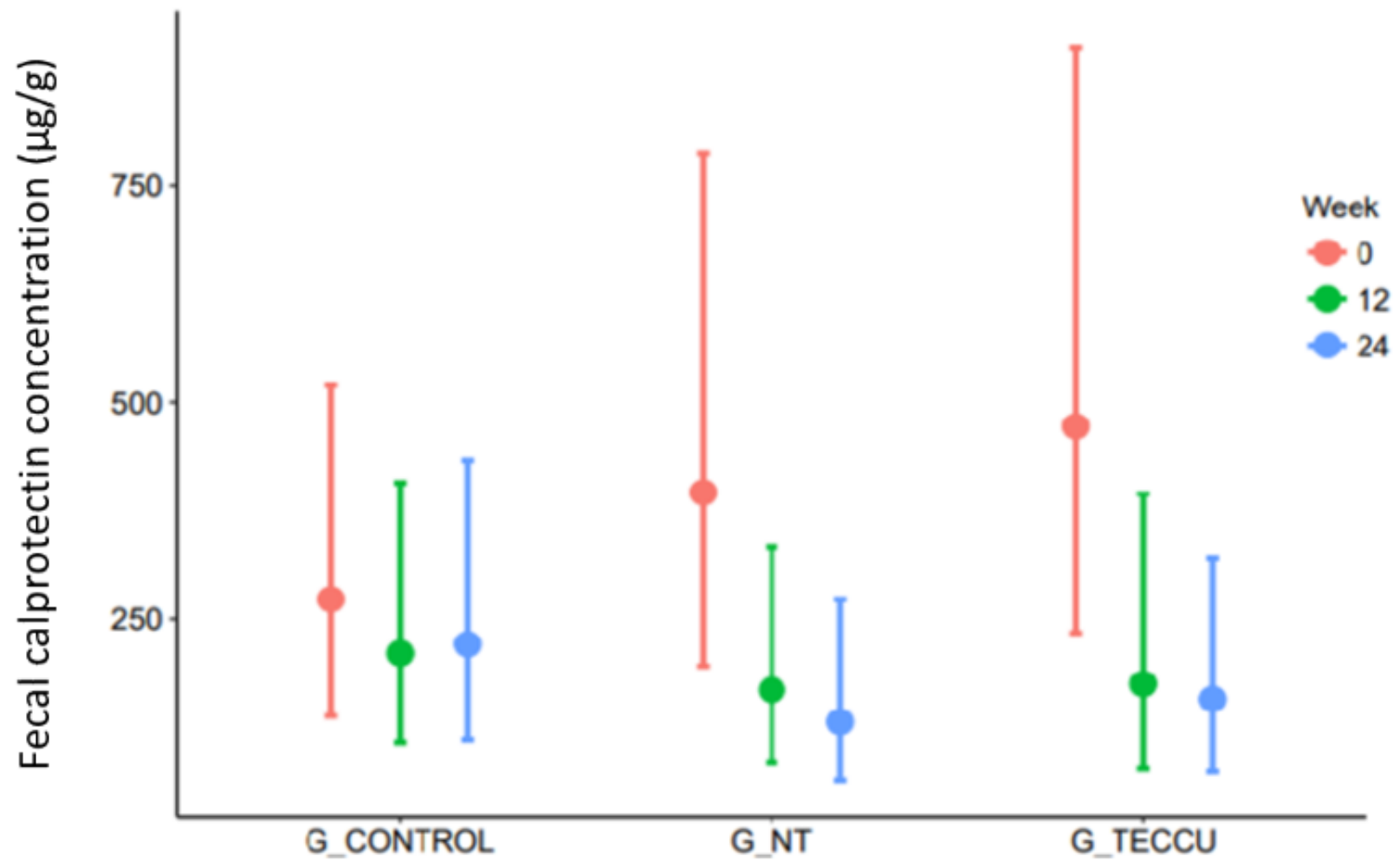

\section{Health-Related Quality of Life}

HRQoL scores were similar between all groups at baseline, and the HRQoL of patients from all 3 groups improved after 24 weeks. The median IBDQ-9 scores increased from 38.5 to 53 in $\mathrm{G}_{-}$control, from 37.5 to 53 in $\mathrm{G} \_\mathrm{NT}$, and from 42 to 52.5 in G_TECCU (overall intervention effect on the IBDQ-9 score: $\mathrm{OR}=8.42,95 \% \mathrm{CI}=3.98-17.81, P<.001)$. The median EQ-5D score improved from 0.816 to $1.00 \mathrm{in} \mathrm{G}$ _control and from 0.825 to 1.00 in G_NT and G_TECCU (overall intervention effect on the EQ-5D score: $\mathrm{OR}=1.99,95 \% \mathrm{CI}=1.09-3.63, P<.001)$. However, the improvement in HRQoL was not significantly different among groups with regard to the IBDQ-9 score
(G_TECCU vs G_control: $\mathrm{OR}=1.25,95 \% \mathrm{CI}=0.49-3.15, P=.64$; G_NT vs G_control: $\mathrm{OR}=0.79,95 \% \mathrm{CI}=0.32-1.98, P=.62)$ and the EQ-5D score (G_TECCU vs G_control: OR=1.42, 95\% $\mathrm{CI}=0.49-4.13, P=.52 ; \mathrm{G} \_\mathrm{NT}$ vs $\mathrm{G}_{-}$control: $\mathrm{OR}=1.68,95 \%$ $\mathrm{CI}=0.59-4.81, P=.33)$. In addition, a significant improvement in the HRQoL of patients from the 3 arms was evident in the EQ-5D-VAS scores, with an increase in the median score from $60.5 \%$ to $85 \%$ in G_control, from $62.5 \%$ to $70 \%$ in G_NT, and from $60 \%$ to $80 \%$ in $\mathrm{G}_{-} \mathrm{TECCU}$ (overall intervention effect on the score increase: $\mathrm{OR}=3.53,95 \% \mathrm{CI}=1.79-6.95, P<.001$ ); however, the improvement was not significantly different between the groups at 24 weeks $\left(G_{-}\right.$TECCU vs G_control: $\mathrm{OR}=0.63,95 \% \mathrm{CI}=0.26-1.57, P=.32 ; \mathrm{G} \_\mathrm{NT}$ vs G_control: 
$\mathrm{OR}=0.69,95 \% \mathrm{CI}=0.28-1.71, P=.43$; Figure 6). The HRQoL improved in 4 patients in $\mathrm{G}_{-}$TECCU who were followed up via mobile phone, from a median baseline IBDQ-9 score of 31.5 to 47.75 and a median baseline EQ-5D score of 0.71 to 0.94 , with an improvement in the median EQ-5D-VAS score from $50 \%$ to $70 \%$ at study completion.

\section{Work Productivity and Social Activities}

With regard to work productivity and activity impairment, we compared the median percentage of work hours missed owing to disease on the basis of the answers to question 2 of the WPAI questionnaire ("During the past seven days, how many hours did you miss from work because of problems associated with your ulcerative colitis/Crohn's disease?"). We found wide variability in the percentage of hours missed in the $3 \mathrm{arms}$, with no significant reduction in any group $(\mathrm{OR}=0.99,95 \%$ $\mathrm{CI}=0.43-2.29, P=.93)$ and no differences among the groups at 24 weeks (G_NT vs G_control: $\mathrm{OR}=0.64,95 \% \mathrm{CI}=0.21-1.99$, $P=.44 ; G_{-}$TECCU vs G_control: $\mathrm{OR}=0.97,95 \% \mathrm{CI}=0.31-3.08$, $P=.96)$. Moreover, the median scores of impairment in work productivity over 12 weeks showed a larger reduction in G_TECCU than in $\mathrm{G}_{-}$control $(\mathrm{OR}=0.14,95 \% \mathrm{CI}=0.01-1.46$, $P=.10$; G_NT vs G_control: $\mathrm{OR}=0.13,95 \% \mathrm{CI}=0.01-1.13$, $P=.06)$, but this improvement was not maintained at 24 weeks (G_TECCU vs G_control: $\mathrm{OR}=0.32,95 \% \mathrm{CI}=0.03-2.94, P=.31$; G_AT vs G_control: $\mathrm{OR}=0.21,95 \% \mathrm{CI}=0.03-1.67, P=.14)$. With regard to social impairment, we found a significant improvement in daily activities and a significant reduction in the median score of each group at 12 weeks $(\mathrm{OR}=0.16,95 \%$ $\mathrm{CI}=0.05-0.49, \quad P=.002)$ and 24 weeks $(\mathrm{OR}=0.26,95 \%$ $\mathrm{CI}=0.09-0.77, P=.02)$. This reduction in social impairment was larger in $\mathrm{G}_{-} \mathrm{TECCU}$ than in $\mathrm{G} \_$control at 24 weeks $(\mathrm{OR}=0.27$, 95\% $\mathrm{CI}=0.05-1.49, P=.13$ )

\section{Medication Adherence}

Medication adherence was poorer in G_NT than in the other 2 groups at baseline: $33.3 \%(7 / 21)$ of patients adhered to medication in G_NT; $66.7 \%$ (14/21), in G_control; and 57.1\% $(12 / 21)$, in G_TECCU. Despite these differences, medication adherence improved significantly in the 3 arms: $81 \%(17 / 21)$ in $\mathrm{G}$ _control, $71.4 \%(15 / 21)$ in $\mathrm{G} \_\mathrm{NT}$, and $85.7 \%(18 / 21)$ in G_TECCU at 24 weeks (overall intervention effect on Morisky-Green score reduction: OR=0.051, 95\% $\mathrm{CI}=0.001-0.769)$. In addition, all completers adhered to treatment in G_TECCU (Morisky-Green score=0), and the reduction in the Morisky-Green score was significantly more pronounced in G_TECCU than in $\mathrm{G}$ _control $(\mathrm{OR}=0.0001,95 \%$ $\mathrm{CI}=1.02 \mathrm{e}^{-10}$ to 0.517 ; Figure 7).

\section{Safety Intervention}

The safety of the 3 interventions was similar, and no differences were noted among the groups for visits to the emergency department ( 0 in G_TECCU, 2 in the G_NT, and 1 in the G_control), IBD-related surgeries (1 in each group), hospitalizations ( 2 in $G_{-}$TECCU, 2 in $G_{-}$NT, and 1 in G_control), and corticosteroid courses (4 in G_TECCU, 4 in G_NT, and 3 in G_control). Adverse effects of medication were

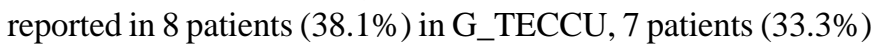
in G_NT, and 9 patients $(42.9 \%)$ in $G_{-}$control after 24 weeks $\left(P=.92\right.$ for $G_{-}$TECCU vs $G_{-}$control). No patients died during the study, and adverse effects related to the follow-up intervention were not reported.

\section{Use of Health Care Resources}

The total number of outpatient visits to the gastroenterologist or nurse after 24 weeks was lower in G_TECCU (72 visits; $25 \%$ of total) and G_NT (85 visits; $29.5 \%$ of total) than in G_control (131 visits; $45.5 \%$ of total). Similarly, the number of telephone calls was lower in G_TECCU (12 calls; $6.8 \%$ of total) than in G_NT (118 calls; $66.7 \%$ of total) and G_control (47 calls; $26.5 \%$ of total).

Figure 6. Evolution of the quality of life over the study period in the 3 groups. G_CONTROL: group receiving standard care with in-person visits; G_NT: group receiving nurse-assisted telephone care; G_TECCU: group receiving remote monitoring; IBDQ-9: Inflammatory Bowel Disease Questionnaire 9 .

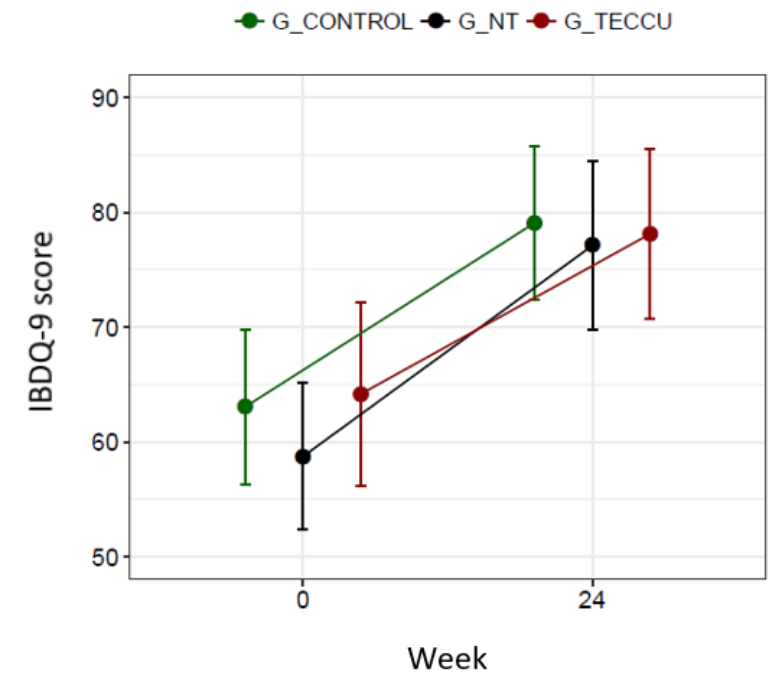

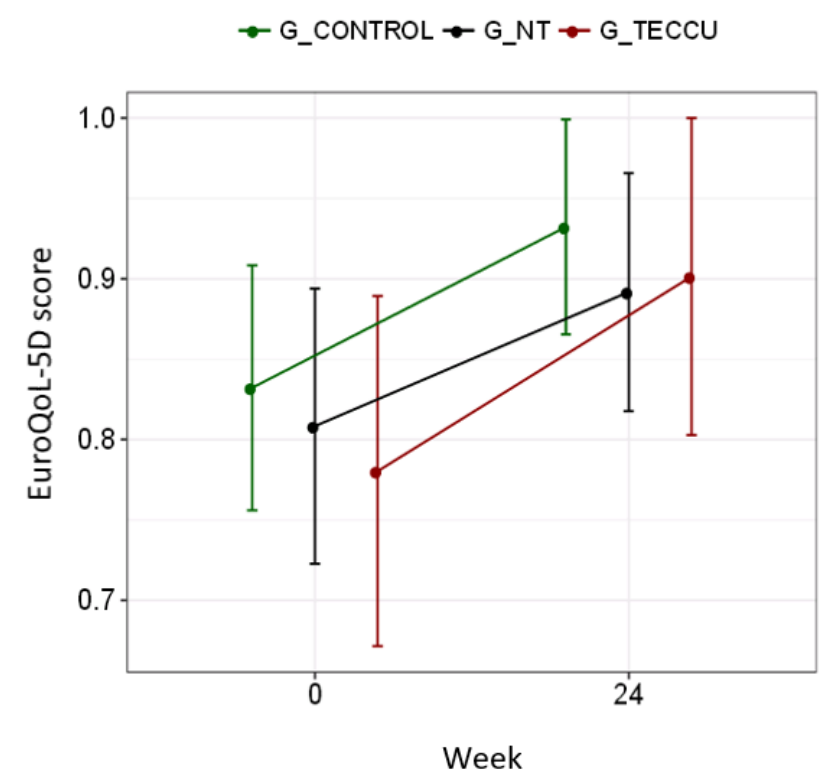


Figure 7. Evolution of the Morisky-Green score over the study period in the 3 groups. G_CONTROL: group receiving standard care with in-person visits; G_NT: group receiving nurse-assisted telephone care; G_TECCU: group receiving remote monitoring.

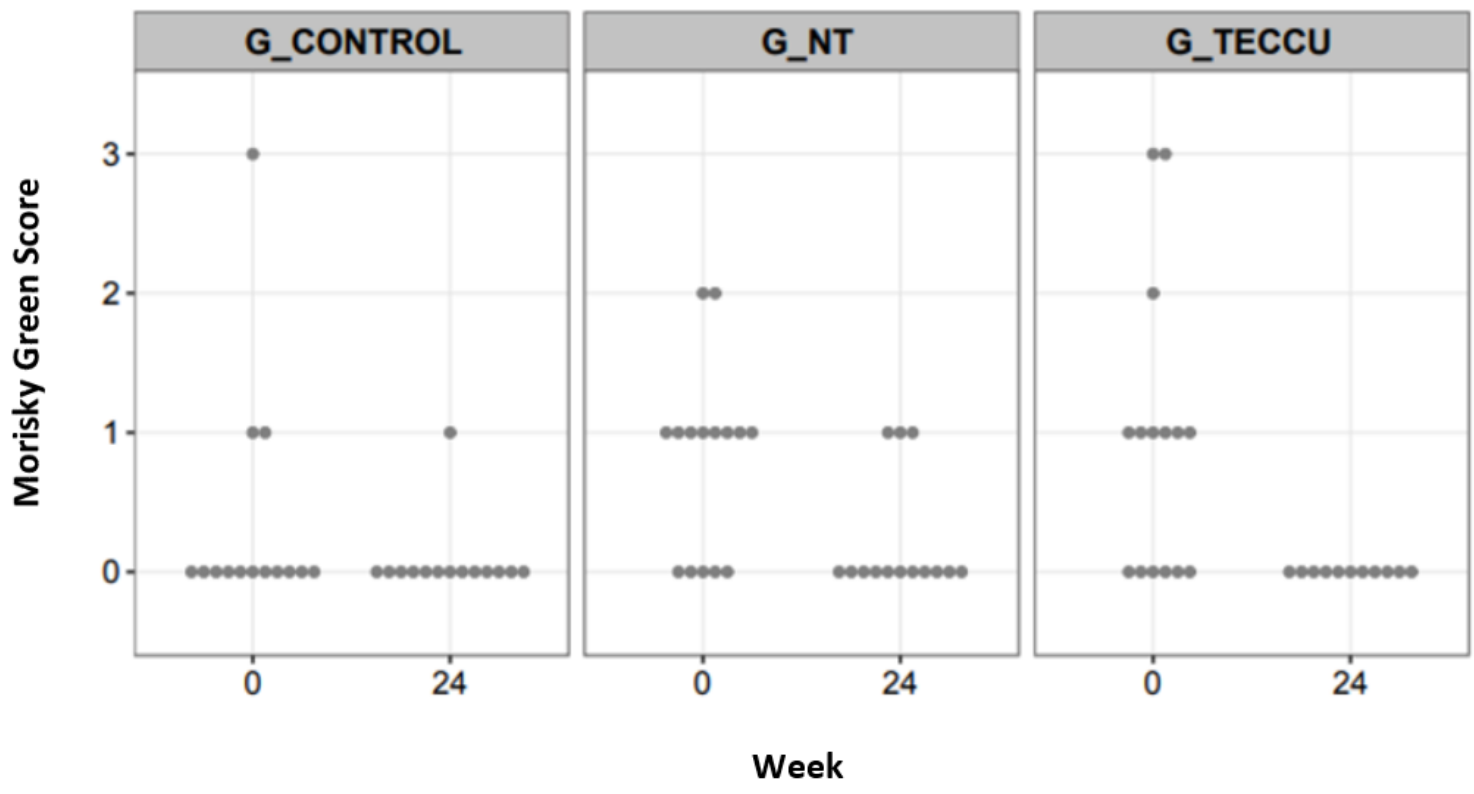

\section{Patient Satisfaction}

Patient satisfaction improved from a median score of 52 to 57 in G_TECCU and from 49.5 to 55 in G_control (overall intervention effect: $\mathrm{OR}=8.93,95 \% \mathrm{CI}=2.97-26.84, P<.001)$ at 24 weeks; however, the satisfaction score remained unchanged at 53 points in G_NT. Satisfaction with previous care was high in the mobile phone subgroup of G_TECCU, with a median score of 54.25 , although this score improved to 56.25 after 24 weeks of follow-up via mobile phone.

In G_TECCU, neither the patients nor the researchers perceived privacy breaches while using the Web platform. Only two patients in G_TECCU reported minor technical problems with temporary unavailability of the webpage over the follow-up period, but technical assistants resolved the issue remotely. The 3 patients who were noncompliant to the follow-up schedule were contacted by telephone, and 2 of them who were insufficiently compliant specified that they forgot to follow some remote controls because they were in acceptable health conditions. We subsequently scheduled in-person visits, but these patients only attended them once. The only patient who was lost to follow-up mentioned that neither the previous standard care nor the Web platform met her expectations; therefore, she decided to move to another center.

All staff, including 5 gastroenterologists and 2 nurses specializing in IBD, showed good acceptance of the platform and considered it easy to use. However, 2 physicians thought that the calendar included in the platform for health care providers to survey the next controls for each patient was not completely intuitive and would benefit from some change in its appearance. Furthermore, 1 physician reported minor technical problems with temporary unavailability of the webpage over the follow-up period, but the technical assistants resolved the issue remotely after 1 day.

\section{Discussion}

\section{Principal Findings}

We performed a randomized controlled clinical trial to compare the impact of a Web-based telemanagement system (TECCU), nurse-assisted telephone care, and standard face-to-face visits on health outcomes and outpatient visits in patients with complex IBD. Patients with IBD who start treatment with systemic corticosteroids, immunosuppressants, and biological agents for control of inflammatory activity are considered to have moderate-to-high complexity of disease. Our results showed that TECCU was safe and effective for improving health outcomes and the use of health care resources in this setting.

\section{Comparison With Prior Work}

Although telemonitoring apps are well accepted and seem to be a safe approach for follow-up of patients in remission or with mild activity $[6,18,41]$, the reported efficacy of telemonitoring on disease activity and QoL is inconsistent between studies $[14,42]$, and no specific trials have evaluated the impact of telemedicine in patients with complex IBD. Therefore, we designed a controlled 3-arm clinical trial to assess the impact of the TECCU Web program on disease outcomes and health care use in comparison with the main strategies applied to date for the follow-up of patients with complex IBD (standard or telephone support provided by a nurse).

This program was designed in collaboration with patients and researchers to address the particular needs of patients with complex IBD, according to national and European clinical guidelines. As detailed elsewhere [27], this information was structured and filtered using an intelligent prioritization system, with generation of alerts and push notifications according to an integrated intervention protocol, which facilitated a rapid response from nurses and physicians for different events occurring during follow-up in a time period according to the severity of each alert. Furthermore, the Web platform allowed continuous communication between patients and health 
providers via electronic messaging, and we incorporated educational elements in the platform to improve disease knowledge among patients and empowerment through interactive materials. With the use of the TECCU Web platform, we found a significant improvement in HRQoL, medication adherence, social activities, and satisfaction; in addition, disease activity improved at the end of follow-up in each group. Moreover, a greater improvement in disease activity and social activities was reported with TECCU than with standard care, but the differences were not significant, probably due to the small sample size and the relatively short follow-up time.

In agreement with previous data [6,8], medication adherence was better in $G_{-}$TECCU compared to the other groups and reached $100 \%$ among completers in G_TECCU, probably because care was continuously adapted at each stage of the disease and communication with physicians was better in this group. Therefore, we were able to easily identify eventual problems related to poor adherence [2]. In addition, constant tailored monitoring with Web TECCU could reduce interference with daily activities, as social impairment due to disease tended to improve in G_TECCU compared with the other two arms. Not surprisingly, the improvement in remission rates and social functioning was associated with high patient satisfaction and correlated directly with a significant improvement in HRQoL, measured by the specific IDBQ-9 and the EQ-5D.

The Web TECCU and nurse-assisted telephone care were associated with fewer outpatient visits than standard care, but the Web-monitoring system reduced the number of telephone calls compared with the other two groups. Our study was performed in a referral hospital with an accredited, well-structured IBD Unit and accessible outpatient clinics with specialized nurses and e-mail or telephone consultations. Therefore, the reduction of face-to-face visits suggests that the effect of the TECCU remote-monitoring system on the frequency of outpatient visits could be favorable in hospitals with referral IBD Units. Although this reduction in office visits could be influenced by the follow-up schedule used in our study, the frequency of visits for all the 3 arms was designed according to the standard clinical practice in our center and based on national and European guidelines. Furthermore, our Web-based telemanagement system was safe and well accepted by patients, with no adverse effects related to the study intervention and no differences among groups in terms of emergency visits, surgeries, hospitalizations, corticosteroid courses, or medication adverse effects, which is consistent with the findings of previous studies $[6,8]$.

Despite different attrition rates in the use of telemedicine apps, our results indicate that it is important to emphasize the feasibility of telemedicine for providing solutions for the medical and social impact of IBD. In our study, only 3 patients did not complete the follow-up schedule in G_TECCU (1 patient was lost to follow-up and 2 patients were insufficiently compliant); this low proportion is likely a result of the reminder system integrated in the platform and the short follow-up period. However, previous clinical trials reported high withdrawal rates in telemedicine groups $[6,8,18,22]$, despite adaptations in the platform design over recent years. Consequently, telemedicine is not suitable for all patients, and improvements in telemonitoring apps and the patient-selection criteria are necessary. Nevertheless, this is not necessarily a barrier to the application of telemedicine, because the majority of patients are adherent to remote monitoring, and the efficacy of these follow-up methods has been reported in different populations including patients with complex IBD, as reported in our trial. It is important to evaluate the disease phenotype, discuss preferences with the patient, and assess the ability to use ICTs. Some patients such as those in the immediate postoperative period and those with perianal disease, who require physical examination, may not benefit from remote monitoring with the technological tools available thus far.

\section{Strengths}

The main strength of our study was its randomized controlled design, which allowed us to evaluate the impact of a Web telemanagement system on disease outcomes and compare the approach with the main strategies applied in the follow-up of IBD patients in daily practice. In addition, allocation concealment ensured that researchers were blinded to group assignment during the randomization process. Furthermore, the selection of patients with complex IBD from a referral center provides important data on the effect of telemedicine in patients with moderate-to-severe disease activity or initiating administration of immunosuppressants and biological agents, which is a specific population that has not been sufficiently represented in previous studies. Another advantage was the selection of the activity index and biological tools for measuring disease activity and other outcomes remotely. The Walmsley index was recently validated for self-administration via an online tool [43] and correlates well with other, more complex-activity indexes based on endoscopy [44]. Similarly, the reduced 6- and 9-point versions of the Mayo index, which are not based on endoscopy, show clinical response to treatment, similar to the full Mayo score [30]. Finally, as HBI may not completely reflect the inflammatory activity of the disease [45], the use of biological markers (C-reactive protein and FC) provided added value to remote monitoring, because they are sufficiently sensitive for detecting mucosal inflammation [46], and FC levels can be easily recorded at home.

\section{Limitations}

Our study was subject to a series of limitations. First, considering the specific study population, the sample was not large enough to detect statistically significant differences in clinical activity and the types of interventions assessed; as such, neither the patients nor the researchers were masked to the intervention. It is possible that the differences estimated among groups in the scarce literature on the impact on health outcomes could interfere with the sample size calculation in this pilot trial, and the lack of blinding could improve the usual attention provided to patients in G_control, which could also attenuate differences in these outcomes among groups. Nonetheless, our results were analyzed by an independent statistician who was blinded to group identification. Second, the selection of patients with complex IBD in a tertiary referral hospital prevented extrapolation of our results to the entire IBD population. Third, we did not perform colonoscopy in all cases, because it increases costs and risks to patients and is not performed routinely in all 
patients during daily practice. Instead, we used biological markers that were highly sensitive for endoscopic activity. Finally, the 24-week follow-up period could be considered a short duration, because both patients and physicians need time to learn how to interact with the platform, thus leading to interference with the efficacy of the Web system in terms of disease outcome in the experimental group. Therefore, trials with longer follow-up periods are required to confirm the efficacy of telemedicine in improving long-term disease outcomes.

\section{Conclusions}

TECCU is a safe tool for the control of disease activity and improves HRQoL, medication adherence, and impairment in social activities in patients with complex IBD. In addition, it reduces the use of health care resources and the number of outpatient visits and telephone calls. It is important to select patients who are willing and able to use Web programs, since the results of this study suggest greater efficacy in health outcomes with a consequent improvement in the HRQoL and satisfaction of patients who adhere to telemanagement. Web-based systems are a safe and feasible option for IBD monitoring and could play a key role in reorganizing the structure of health systems if they prove to be cost-effective in the long-term. On the other hand, although telemonitoring platforms are well accepted, thus far, they have not clearly demonstrated their efficacy on health outcomes in patients with complex IBD and those in remission or with mild-to-moderate disease. Future studies with longer follow-up periods are necessary to confirm our findings in order to determine whether Web-based programs can improve the long-term course of IBD in a more-complex setting.

\section{Acknowledgments}

This study was supported by grants from the Instituto de Salud Carlos III-Fondo de Investigaciones Sanitarias (FIS PI12/00277) and cofunded by FEDER (Fondo Europeo de Desarrollo Regional).

\section{Conflicts of Interest}

DD is the general manager of Connected Health Services.

\section{Multimedia Appendix 1 \\ CONSORT-EHEALTH checklist (V 1.6.1).}

[PDF File (Adobe PDF File), 511KB-Multimedia Appendix 1]

\section{References}

1. Kappelman MD, Porter CQ, Galanko JA, Rifas-Shiman SL, Ollendorf DA, Sandler RS, et al. Utilization of healthcare resources by U.S. children and adults with inflammatory bowel disease. Inflamm Bowel Dis 2011 Jan;17(1):62-68 [FREE Full text] [doi: 10.1002/ibd.21371] [Medline: 20564532]

2. Jackson CA, Clatworthy J, Robinson A, Horne R. Factors associated with non-adherence to oral medication for inflammatory bowel disease: a systematic review. Am J Gastroenterol 2010 Mar;105(3):525-539. [doi: 10.1038/ajg.2009.685] [Medline: 19997092]

3. van Deen WK, van Oijen MGH, Myers KD, Centeno A, Howard W, Choi JM, et al. A nationwide 2010-2012 analysis of U.S. health care utilization in inflammatory bowel diseases. Inflamm Bowel Dis 2014 Oct;20(10):1747-1753. [doi: 10.1097/MIB.0000000000000139] [Medline: 25137415]

4. Høivik ML, Moum B, Solberg IC, Henriksen M, Cvancarova M, Bernklev T, IBSEN Group. Work disability in inflammatory bowel disease patients 10 years after disease onset: results from the IBSEN Study. Gut 2013 Mar;62(3):368-375. [doi: 10.1136/gutjnl-2012-302311] [Medline: 22717453]

5. Hoivik ML, Moum B, Solberg IC, Cvancarova M, Hoie O, Vatn MH, IBSEN Study Group. Health-related quality of life in patients with ulcerative colitis after a 10-year disease course: results from the IBSEN study. Inflamm Bowel Dis 2012 Aug;18(8):1540-1549. [doi: 10.1002/ibd.21863] [Medline: 21936030]

6. Elkjaer M, Shuhaibar M, Burisch J, Bailey Y, Scherfig H, Laugesen B, et al. E-health empowers patients with ulcerative colitis: a randomised controlled trial of the web-guided 'Constant-care' approach. Gut 2010 Dec;59(12):1652-1661. [doi: 10.1136/gut.2010.220160] [Medline: 21071584]

7. Krier M, Kaltenbach T, McQuaid K, Soetikno R. Potential use of telemedicine to provide outpatient care for inflammatory bowel disease. Am J Gastroenterol 2011 Dec;106(12):2063-2067. [doi: 10.1038/ajg.2011.329] [Medline: 22138934]

8. de Jong MJ, van der Meulen-de Jong AE, Romberg-Camps MJ, Becx MC, Maljaars JP, Cilissen M, et al. Telemedicine for management of inflammatory bowel disease (myIBDcoach): a pragmatic, multicentre, randomised controlled trial. Lancet 2017 Sep 02;390(10098):959-968. [doi: 10.1016/S0140-6736(17)31327-2] [Medline: 28716313]

9. Pedersen N, Elkjaer M, Duricova D, Burisch J, Dobrzanski C, Andersen NN, et al. eHealth: individualisation of infliximab treatment and disease course via a self-managed web-based solution in Crohn's disease. Aliment Pharmacol Ther 2012 Nov;36(9):840-849 [FREE Full text] [doi: 10.1111/apt.12043] [Medline: 22971016] 
10. Pedersen N, Thielsen P, Martinsen L, Bennedsen M, Haaber A, Langholz E, et al. eHealth: individualization of mesalazine treatment through a self-managed web-based solution in mild-to-moderate ulcerative colitis. Inflamm Bowel Dis 2014 Dec;20(12):2276-2285. [doi: 10.1097/MIB.0000000000000199] [Medline: 25248002]

11. Clarke M, Shah A, Sharma U. Systematic review of studies on telemonitoring of patients with congestive heart failure: a meta-analysis. J Telemed Telecare 2011;17(1):7-14. [doi: 10.1258/jtt.2010.100113] [Medline: 21097564]

12. Weinstock RS, Teresi JA, Goland R, Izquierdo R, Palmas W, Eimicke JP, et al. Glycemic control and health disparities in older ethnically diverse underserved adults with diabetes: five-year results from the Informatics for Diabetes Education and Telemedicine (IDEATel) study. Diabetes Care 2011 Feb;34(2):274-279 [FREE Full text] [doi: 10.2337/dc10-1346] [Medline: 21270184]

13. Bartoli L, Zanaboni P, Masella C, Ursini N. Systematic review of telemedicine services for patients affected by chronic obstructive pulmonary disease (COPD). Telemed J E Health 2009 Nov;15(9):877-883. [doi: 10.1089/tmj.2009.0044] [Medline: 19919194]

14. Aguas PM, Del HJ, Bebia P, Faubel R, Barrios A, Bastida G, et al. Telemedicine in inflammatory bowel disease: opportunities and approaches. Inflamm Bowel Dis 2015 Feb;21(2):392-399. [doi: 10.1097/MIB.0000000000000241] [Medline: 25437818]

15. Helsel BC, Williams JE, Lawson K, Liang J, Markowitz J. Telemedicine and Mobile Health Technology Are Effective in the Management of Digestive Diseases: A Systematic Review. Dig Dis Sci 2018 Jun;63(6):1392-1408. [doi:

10.1007/s10620-018-5054-z] [Medline: 29663265]

16. Cross RK, Cheevers N, Finkelstein J. Home telemanagement for patients with ulcerative colitis (UC HAT). Dig Dis Sci 2009 Nov;54(11):2463-2472. [doi: 10.1007/s10620-008-0640-0] [Medline: 19104937]

17. Cross RK, Arora M, Finkelstein J. Acceptance of telemanagement is high in patients with inflammatory bowel disease. J Clin Gastroenterol 2006 Mar;40(3):200-208. [Medline: 16633120]

18. Cross RK, Cheevers N, Rustgi A, Langenberg P, Finkelstein J. Randomized, controlled trial of home telemanagement in patients with ulcerative colitis (UC HAT). Inflamm Bowel Dis 2012 Jun;18(6):1018-1025 [FREE Full text] [doi: 10.1002/ibd.21795] [Medline: 21688350]

19. Cross RK, Jambaulikar G, Langenberg P, Tracy JK, Collins JF, Katz J, et al. TELEmedicine for Patients with Inflammatory Bowel Disease (TELE-IBD): Design and implementation of randomized clinical trial. Contemp Clin Trials 2015 May;42:132-144. [doi: 10.1016/j.cct.2015.03.006] [Medline: 25812483]

20. Carlsen K, Jakobsen C, Houen G, Kallemose T, Paerregaard A, Riis LB, et al. Self-managed eHealth Disease Monitoring in Children and Adolescents with Inflammatory Bowel Disease: A Randomized Controlled Trial. Inflamm Bowel Dis 2017 Dec;23(3):357-365. [doi: 10.1097/MIB.0000000000001026] [Medline: 28221247]

21. Carlsen K, Houen G, Jakobsen C, Kallemose T, Paerregaard A, Riis LB, et al. Individualized Infliximab Treatment Guided by Patient-managed eHealth in Children and Adolescents with Inflammatory Bowel Disease. Inflamm Bowel Dis 2017 Dec;23(9):1473-1482. [doi: 10.1097/MIB.0000000000001170] [Medline: 28617758]

22. Heida A, Dijkstra A, Muller KA, Rossen JW, Kindermann A, Kokke F, et al. Efficacy of Home Telemonitoring versus Conventional Follow-up: A Randomized Controlled Trial among Teenagers with Inflammatory Bowel Disease. J Crohns Colitis 2018 Mar 28;12(4):432-441. [doi: 10.1093/ecco-jcc/jix169] [Medline: 29228230]

23. Casellas-Jordá F, Borruel-Sainz N, Torrejón-Herrera A, Castells I. Effect upon hospital activity of the application of a continued care model centered on patients with inflammatory bowel disease. Rev Esp Enferm Dig 2012 Feb;104(1):16-20. [Medline: 22300112]

24. Casey M, Hayes PS, Heaney D, Dowie L, Ólaighin G, Matero M, et al. Implementing transnational telemedicine solutions: a connected health project in rural and remote areas of six Northern Periphery countries Series on European collaborative projects. Eur J Gen Pract 2013 Mar;19(1):52-58. [doi: 10.3109/13814788.2012.761440] [Medline: 23432039]

25. Van Assche G, Dignass A, Panes J, Beaugerie L, Karagiannis J, Allez M, European Crohn's Colitis Organisation (ECCO). The second European evidence-based Consensus on the diagnosis and management of Crohn's disease: Definitions and diagnosis. J Crohns Colitis 2010 Feb;4(1):7-27 [FREE Full text] [doi: 10.1016/j.crohns.2009.12.003] [Medline: 21122488]

26. Dignass A, Eliakim R, Magro F, Maaser C, Chowers Y, Geboes K, et al. Second European evidence-based consensus on the diagnosis and management of ulcerative colitis part 1: definitions and diagnosis. J Crohns Colitis 2012 Dec;6(10):965-990 [FREE Full text] [doi: 10.1016/j.crohns.2012.09.003] [Medline: 23040452]

27. Aguas M, Del Hoyo J, Faubel R, Muñoz D, Domínguez D, Bastida G. A web-based telemanagement system for patients with complex inflammatory bowel disease: protocol for a randomized controlled clinical trial. JMIR Res Protoc 2018 Dec:1-12. [doi: 10.2196/resprot.9639]

28. Harvey RF, Bradshaw JM. A simple index of Crohn's-disease activity. Lancet 1980 Mar 8;1(8167):514. [Medline: 6102236]

29. Walmsley RS, Ayres RC, Pounder RE, Allan RN. A simple clinical colitis activity index. Gut 1998 Jul;43(1):29-32 [FREE Full text] [Medline: 9771402]

30. Lewis JD, Chuai S, Nessel L, Lichtenstein GR, Aberra FN, Ellenberg JH. Use of the noninvasive components of the Mayo score to assess clinical response in ulcerative colitis. Inflamm Bowel Dis 2008 Dec;14(12):1660-1666 [FREE Full text] [doi: 10.1002/ibd.20520] [Medline: 18623174] 
31. D'Haens G, Sandborn WJ, Feagan BG, Geboes K, Hanauer SB, Irvine EJ, et al. A review of activity indices and efficacy end points for clinical trials of medical therapy in adults with ulcerative colitis. Gastroenterology 2007 Feb;132(2):763-786. [doi: 10.1053/j.gastro.2006.12.038] [Medline: 17258735]

32. Alcalá MJ, Casellas F, Fontanet G, Prieto L, Malagelada J. Shortened questionnaire on quality of life for inflammatory bowel disease. Inflamm Bowel Dis 2004 Jul;10(4):383-391. [Medline: 15475746]

33. Badia X, Roset M, Montserrat S, Herdman M, Segura A. [The Spanish version of EuroQol: a description and its applications. European Quality of Life scale]. Med Clin (Barc) 1999;112 Suppl 1:79-85. [Medline: 10618804]

34. Badia X, Roset M, Herdman M, Kind P. A comparison of United Kingdom and Spanish general population time trade-off values for EQ-5D health states. Med Decis Making 2001;21(1):7-16. [doi: 10.1177/0272989X0102100102] [Medline: 11206949]

35. Vergara M, Montserrat A, Casellas F, Villoria A, Suarez D, Maudsley M, et al. A new validation of the Spanish Work Productivity and Activity Impairment Questionnaire-Crohn's disease version. Value Health 2011;14(6):859-861 [FREE Full text] [doi: 10.1016/j.jval.2011.02.1179] [Medline: 21914506]

36. Morisky DE, Green LW, Levine DM. Concurrent and predictive validity of a self-reported measure of medication adherence. Med Care 1986 Jan;24(1):67-74. [Medline: 3945130]

37. Larsen DL, Attkisson CC, Hargreaves WA, Nguyen TD. Assessment of client/patient satisfaction: development of a general scale. Eval Program Plann 1979;2(3):197-207. [Medline: 10245370]

38. TECCU. URL: http://82.223.37.103/teccu [accessed 2018-11-03] [WebCite Cache ID 73eLybxRj]

39. Gomollón F, García-López S, Sicilia B, Gisbert JP, Hinojosa J, en representación del Grupo Español de Trabajo de Enfermedad de Crohn y Colitis Ulcerosa or Spanish Group for Working on Crohn'sDiseaseUlcerative Colitis (GETECCU). [Therapeutic guidelines on ulcerative colitis: a GRADE methodology based effort of GETECCU]. Gastroenterol Hepatol 2013 Oct;36(8):e1-47. [Medline: 24215088]

40. R Core Team (2013). R: A language and environment for statistical computing. Vienna, Austria: R Foundation for Statistical Computing URL: http://www.r-project.org/ [accessed 2018-11-07] [WebCite Cache ID 73knzJR3d]

41. Robinson A, Thompson DG, Wilkin D, Roberts C, Northwest GRG. Guided self-management and patient-directed follow-up of ulcerative colitis: a randomised trial. Lancet 2001 Sep 22;358(9286):976-981. [doi: 10.1016/S0140-6736(01)06105-0] [Medline: 11583752$]$

42. Aguas M, Del HJ, Faubel R, Valdivieso B, Nos P. Telemedicine in the treatment of patients with inflammatory bowel disease. Gastroenterol Hepatol 2017 Nov;40(9):641-647. [doi: 10.1016/j.gastrohep.2017.07.001] [Medline: 28797518]

43. Marín-Jiménez I, Nos P, Domènech E, Riestra S, Gisbert JP, Calvet X, et al. Diagnostic Performance of the Simple Clinical Colitis Activity Index Self-Administered Online at Home by Patients With Ulcerative Colitis: CRONICA-UC Study. Am J Gastroenterol 2016 Feb;111(2):261-268. [doi: 10.1038/ajg.2015.403] [Medline: 26753886]

44. Higgins PDR, Schwartz M, Mapili J, Zimmermann EM. Is endoscopy necessary for the measurement of disease activity in ulcerative colitis? Am J Gastroenterol 2005 Feb;100(2):355-361. [doi: 10.1111/j.1572-0241.2005.40641.x] [Medline: 15667493]

45. Zittan E, Kabakchiev B, Kelly OB, Milgrom R, Nguyen GC, Croitoru K, et al. Development of the Harvey-Bradshaw Index-pro (HBI-PRO) Score to Assess Endoscopic Disease Activity in Crohn's Disease. J Crohns Colitis 2017 May 01;11(5):543-548. [doi: 10.1093/ecco-jcc/jjw200] [Medline: 28453763]

46. Lin J, Chen J, Zuo J, Yu A, Xiao Z, Deng F, et al. Meta-analysis: fecal calprotectin for assessment of inflammatory bowel disease activity. Inflamm Bowel Dis 2014 Aug;20(8):1407-1415. [doi: 10.1097/MIB.0000000000000057] [Medline: 24983982]

\author{
Abbreviations \\ CD: Crohn disease \\ EQ-5D: EuroQol-5D \\ FC: fecal calprotectin \\ G_control: group receiving standard care with in-person visits \\ G_NT: group receiving nurse-assisted telephone care \\ G_TECCU: group receiving remote monitoring \\ HBI: Harvey-Bradshaw index \\ HRQoL: health-related quality of life \\ IBD: inflammatory bowel disease \\ IBDQ-9: Inflammatory Bowel Disease Questionnaire 9 \\ ICT: information and communication technologies \\ SCCAI: Simple Clinical Colitis Activity Index \\ TECCU: Telemonitoring of Crohn's Disease and Ulcerative Colitis \\ UC: ulcerative colitis \\ VAS: visual analog scale
}


WPAI: Work Productivity and Activity Impairment

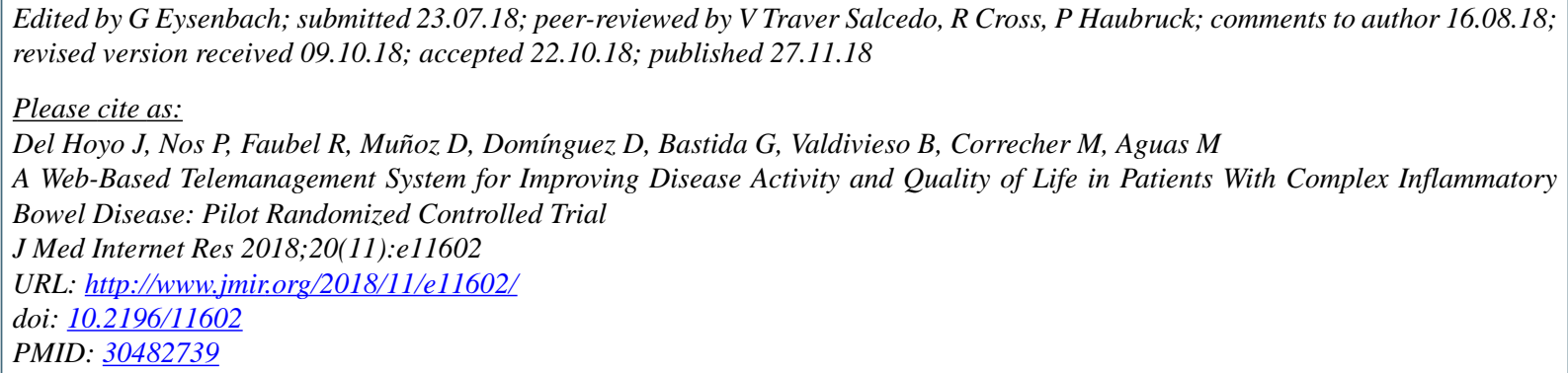

CJavier Del Hoyo, Pilar Nos, Raquel Faubel, Diana Muñoz, David Domínguez, Guillermo Bastida, Bernardo Valdivieso, Marisa Correcher, Mariam Aguas. Originally published in the Journal of Medical Internet Research (http://www.jmir.org), 27.11.2018. This is an open-access article distributed under the terms of the Creative Commons Attribution License (https://creativecommons.org/licenses/by/4.0/), which permits unrestricted use, distribution, and reproduction in any medium, provided the original work, first published in the Journal of Medical Internet Research, is properly cited. The complete bibliographic information, a link to the original publication on http://www.jmir.org/, as well as this copyright and license information must be included. 